\title{
An investigation into the wavelength stability of polymer optical fibre Bragg gratings
}

\author{
W. Zhang*a , A. Abang ${ }^{\mathrm{a}}$, D. J. Webb ${ }^{\mathrm{a}}$, and G. -D. Peng ${ }^{\mathrm{b}}$ \\ ${ }^{a}$ Aston Institute of Photonic Technologies, Aston University, Birmingham, B4 7ET, UK \\ ${ }^{\mathrm{b}}$ School of Electrical Engineering and Telecommunications, University of New South Wales, \\ Sydney, NSW 2052, Australia
}

\begin{abstract}
The inscription of Bragg gratings has been demonstrated in PMMA-based polymer optical fibre. The water affinity of PMMA can introduce significant wavelength change in a polymer optical fibre Bragg grating (POFBG). In polymer optical fibre losses are much higher than with silica fibre. Very strong absorption bands related to higher harmonics of vibrations of the $\mathrm{C}-\mathrm{H}$ bond dominate throughout the visible and near infrared. Molecular vibration in substances generates heat, which is referred to as the thermal effect of molecular vibration. This means that a large part of the absorption of optical energy in those spectral bands will convert into thermal energy, which eventually drives water content out of the polymer fibre and reduces the wavelength of POFBG. In this work we have investigated the wavelength stability of POFBGs in different circumstances. The experiment has shown that the characteristic wavelength of a POFBG starts decreasing after a light source is applied to it. This decrease continues until equilibrium inside the fibre is established, depending on the initial water content inside the fibre, the surrounding humidity, the optical power applied, and the fibre size. Our investigation has shown that POFBGs operating at around $850 \mathrm{~nm}$ show much smaller wavelength reduction than those operating at around $1550 \mathrm{~nm}$ in the same fibre; POFBGs with different diameters show different changes; POFBGs powered by a low level light source, or operating in a very dry environment are least affected by this thermal effect.
\end{abstract}

Key words: Polymer optical fibre, PMMA, fibre Bragg grating, humidity, optical absorption, molecular vibration, thermal effect, polymer swelling

\section{INTRODUCTION}

Polymer optical fibres (POF) also referred to as plastic optical fibers, have been available for several decades. However their use has been overshadowed in the last two decades by the success of silica optical fibres. In general, POFs provide a much lower cost alternative to silica optical fibers, despite their high propagation loss. Due to the ease of use POFs have thus been applied for data transmission over short distances. Recent technological advances have helped promote POF as a lower cost alternative to glass fibre or copper at short distances and bit rates of up to $10 \mathrm{Gbps}$ [1]. As sensors, POFs have additional advantages, including high strain limits, high fracture toughness, high flexibility in bending, high sensitivity to strain, large negative thermo-optic coefficients and for some material an affinity for water. Polymers also have excellent compatibility with organic materials, giving them great potential for biomedical applications.

In the last decade polymer optical fibres have found more applications in the sensing area as fibre Bragg gratings (FBGs) have been inscribed into step index [2] and microstructured POF [3] based on PMMA. Polymer optical fibre Bragg gratings (POFBGs) in such fibre were found to be sensitive to relative humidity, temperature and strain. This is in contrast to glass fibre FBGs which do not show any intrinsic humidity sensitivity. Generally, polymers absorb a certain amount of water depending on their kind. Water transportation in amorphous glassy polymers such as PMMA takes place with a dual mechanism. Some water enters into the polymer network and causes the swelling of the polymer, and some accommodates into pre-existing micro voids. It is known that water acts as a polar plasticizer for several organic polymers. The plasticizing effects of water in polymers are completely reversible when the structure of the polymer remains unchanged and chemical transformations are insignificant [4]. The swelling causes volume expansion of the polymer material, which may be reversible in nature and is related to the phenomena of wetting (sorption) and drying (desorption) [5].

*w.zhang@aston.ac.uk

Microstructured and Specialty Optical Fibres, edited by Kyriacos Kalli, Alexis Mendez,

Proc. of SPIE Vol. 8426, 842619 - (C) 2012 SPIE · CCC code: 0277-786X/12/\$18 · doi: 10.1117/12.922306

Proc. of SPIE Vol. $8426842619-1$ 
The affinity for water of PMMA, leads to a swelling of the fibre and an increase of refractive index, both of which contribute to an increase in the Bragg wavelength of a FBG written in the fibre [6]. This is a potentially very useful property, which has possible applications in chemical processing, agriculture, food storage, paper manufacturing, semiconductor and pharmaceutical industries, where humidity is monitored and controlled to ensure product quality. POFBGs have recently been applied, for example, to quantifying the small amount of water present in aviation fuel [7]. More and more applications of POFBGs have been exploited and realized. As a result the characteristic performance of POFBG has attracted more research attention.

\section{CHARACTERISTIC WAVELENGTH OF POFBG}

The Bragg wavelength of a fibre grating depends on the effective core index, $n_{\text {eff, }}$, and the grating pitch, $\Lambda$, both of which depend on the temperature $T$ and the water content $w$ in the case of a POFBG:

$$
\lambda_{\text {Bragg }}=2 n_{\text {eff }}(T, w) \Lambda(T, w)
$$

For constant temperature the Bragg wavelength change is directly related to the water content inside the fibre. Following references $[6,8]$ we assume that the Bragg wavelength shifts linearly with water content in the fibre. Therefore the Bragg wavelength can then be expressed in terms of

$$
\lambda_{B}=\lambda_{B o}+c w,
$$

where $\lambda_{B 0}$, is the initial Bragg wavelength, $c$, a coefficient associated with PMMA swelling, water dependence of the refractive index and swelling induced stress. A sudden change of environmental condition could lead to a water content change in the PMMA, which is a function of time. This process of water sorption or desorption in PMMA can be described by the diffusion theory of mass transfer [9], and this change introduces a change in the characteristic wavelength of POFBG, which can be used for sensing temperature, humidity, water in fuel, and solution concentration. However, the water content in fibre can be affected by either external change or internal change, for instance, caused by the absorption of optical power in the fibre.

In POFs, the primary sources of absorption are vibrations and electronic transitions of molecular groups. This is an inherent property and can vary with type of materials. In view of the fact that a POF's core is made of organic materials (polymers), vibrations of molecular groups such as carbon-hydrogen $(\mathrm{C}-\mathrm{H})$, carbon-deuterium $(\mathrm{C}-\mathrm{D})$, carbon-fluorine $(\mathrm{C}-\mathrm{F})$, carbon-chlorine $(\mathrm{C}-\mathrm{Cl})$, carbon-bromine $(\mathrm{C}-\mathrm{Br})$, oxygen-hydrogen $(\mathrm{O}-\mathrm{H})$, carbon double-bond oxygen $(\mathrm{C}=\mathrm{O})$, carbon-oxygen $(\mathrm{C}-\mathrm{O})$, and carbon-carbon $(\mathrm{C}-\mathrm{C})$ are the fundamental origin of the absorption loss [10]. The fundamental vibration absorption of the molecular bonds mentioned above falls in the infrared region but has absorption overtones extending up to the visible region causing the high optical loss of POFs. Therefore, in organic polymer optical fibres vibrational absorption appears as a major loss source compared to inorganic glass optical fibres. It is well known that molecular vibration in substances generates heat, which is referred to as the thermal effect of molecular vibration. This means that a large part of the absorption of optical energy in those spectral bands will convert into thermal energy. The generated heat drives the water out of the fibre so that the corresponding wavelength of the POFBG varies with the generated heat, i.e., the absorbed optical energy.

A POFBG becomes operational once the light beam is launched into the fibre. Therefore the absorption starts from the light source switching on and the water content in the fibre may begin varying due to the absorption generated heat, leading to a wavelength change of the POFBG over operation time. The following figure shows the time responses of two POFBGs starting from the switch-on of the light source. From it one can readily notice that once the light source is on the wavelengths of the POFBGs start dropping rapidly. The wavelengths eventually get stabilized after some time. However, the stabilization times and the wavelength changes from the initial values for these two gratings are quite different. The POFBG with an initial wavelength of $\sim 1567 \mathrm{~nm}$ takes over 40 minutes to get stabilized and shows a wavelength change of $0.32 \mathrm{~nm}$. The other POFBG shows almost $1 \mathrm{~nm}$ wavelength change and gets stabilized after 1 hour. Since the wavelength change can be so different, it is important to understand the mechanism causing these changes. 


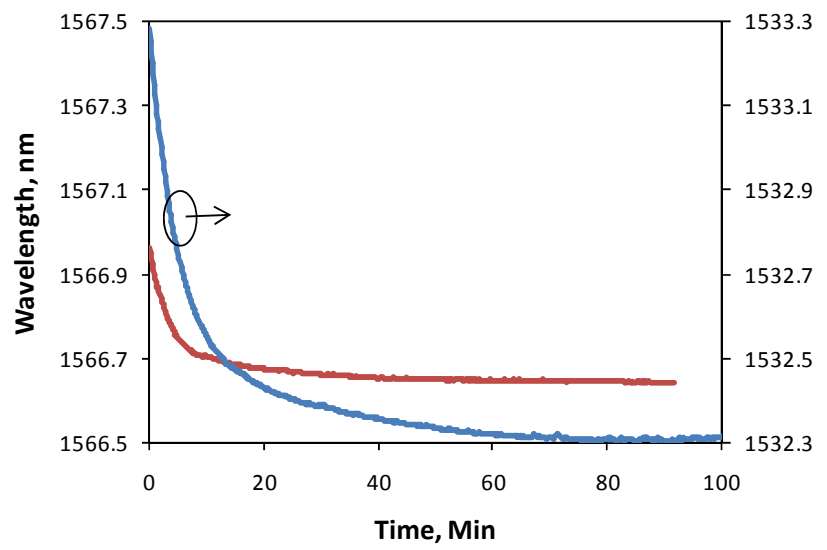

Fig. 1 The time responses of two POFBGs showing the wavelength changes from switch-on of the interrogating light source.

\section{EXPERIMENTS}

The time responses showing in Fig. 1 were measured by using the set-up as shown in Fig. 2. POFBGs were fabricated by attaching a several centimeter length of POF to a single-mode silica fibre down-lead using UV curable glue (Norland 76). The PMMA based POF contained a $5 \mathrm{~mm}$ long FBG, fabricated by illuminating from above a phase mask placed on top of the POF using $325 \mathrm{~nm}$ UV light from a HeCd laser. A broadband light source with a total output optical power of 10 $\mathrm{mW}$ was used, launching a light beam via a fibre circulator, into the POFBG under investigation. The reflection of the POF grating was monitored by using an IBSEN I-MON 400 wavelength interrogation system. The inset shows the captured spectrum of one of the POFBGs. During the experiments the POFBGs were placed inside an environmental chamber (Sanyo Gallenkamp) to allow operation at a desired temperature and humidity.

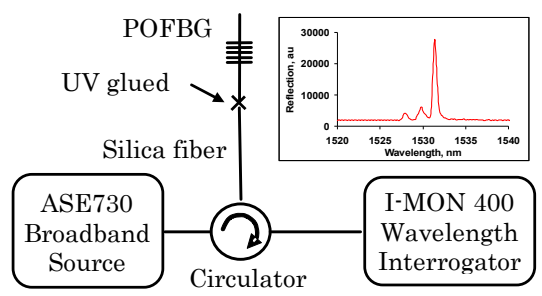

Fig. 2 Experimental arrangement

\subsection{Influence of input optical power}

As described earlier, optical absorption in polymer optical fibre can be converted to thermal energy thus driving the water content out of the fibre. More input optical power means more converted thermal energy, which drives more water out of fibre and causes a greater drop of POFBG wavelength.

POFBGs were tested under different input optical powers to investigate the time response of grating wavelength. A fibre attenuator was inserted in between the broadband source and the circulator to adjust the input optical power to the POFBG. The input optical power can be monitored at port 2 of the fibre circulator. It, however, should be pointed out that this measured power is only the power exiting from the port 2 of the circulator rather than the received power by the polymer optical fibre, since the UV glued joint introduces a significant insertion loss. In the experiment we chose to measure the reflected power from the grating to represent the received optical power by the polymer optical fibre containing the grating.

POFBGs were placed in the environmental chamber while its temperature was set at $22{ }^{\circ} \mathrm{C}$ and the relative humidity at $45 \%$. The wavelength interrogation system (IBSEN I-MON 400) started to record the POFBG wavelength from the 
switch-on of the broadband light source. The time responses of the POFBGs at different input optical power levels were measured. The time responses of the relative wavelength changes of the POFBG with an outer diameter of $135 \mu \mathrm{m}$ are plotted in Fig. 3. One can clearly see that the POFBG wavelength takes different time to get stabilized at different power levels. The final wavelength change is proportional to the input optical power level. The wavelength change against the peak reflection of the POFBG at different input power levels is shown in Fig. 4, illustrating such a trend.

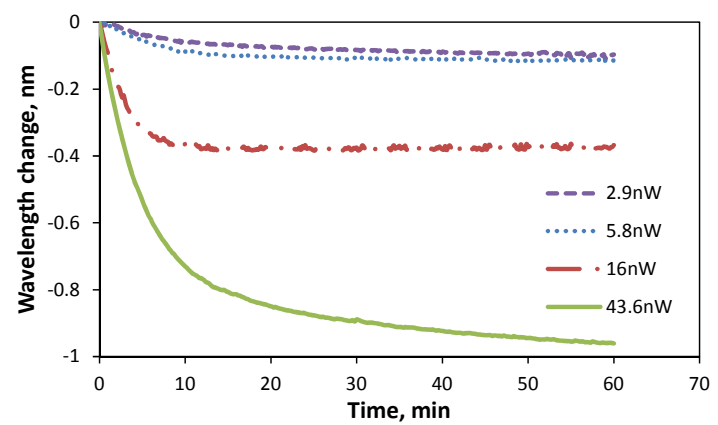

Fig. 3 The time responses of a POFBG wavelength at different input optical power levels.

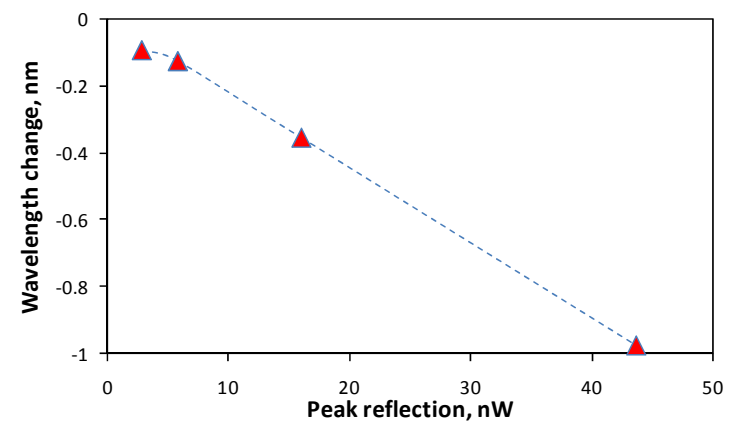

Fig. 4 The wavelength changes vs. the peak reflections of the POFBG

\subsection{Performance at different relative humidities}

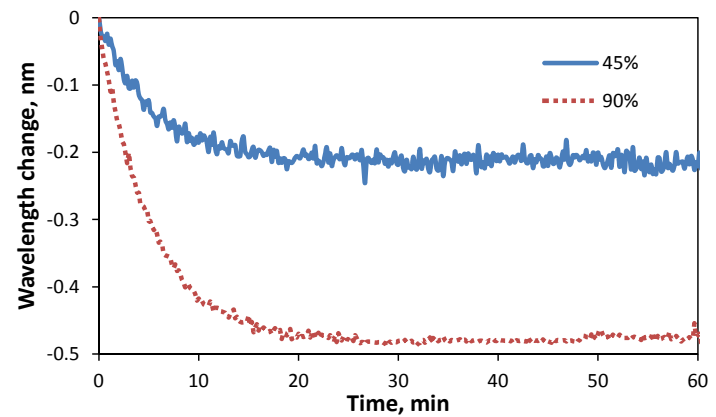

Fig. 5 Typical time responses of POFBG wavelength at different humidity.

According to equation (2) the Bragg wavelength of a POFBG is proportional to the water content in the fibre. The water content in polymer optical fibre depends on the relative humidity of the surrounding. Higher humidity means more water content in the fibre. It consequently gives rise to a larger permeability coefficient for water due to absorbed water induced plasticizing effect on polymer materials [4], which leads to a larger water transportation rate between the fibre and the surrounding. It implies that a larger wavelength change of the POFBG at a higher humidity could be expected for the same input optical power. 
The same POFBG was placed in the environmental chamber with the temperature set to $22{ }^{\circ} \mathrm{C}$ and the humidity set to different values while the input optical power was set with a measured POFBG peak reflection of $16 \mathrm{nW}$. In the experiments the minimum humidity the environmental chamber could reach was $20 \%$. Some typical time responses of the POFBG are shown in Fig. 5.The measured wavelength changes at different humidity settings were plotted in Fig. 6. It shows that for the same input optical power the POFBG wavelength experiences a larger change at a higher relative humidity.

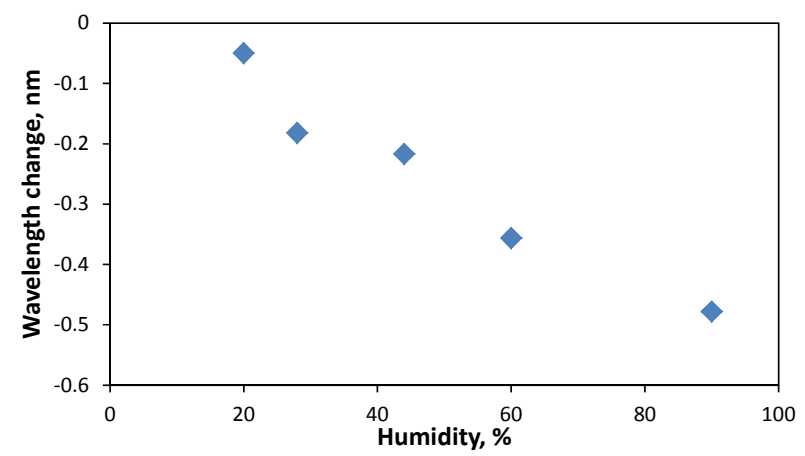

Fig. 6 The wavelength changes vs. the environmental humidity

\subsection{Size of polymer optical fibres}

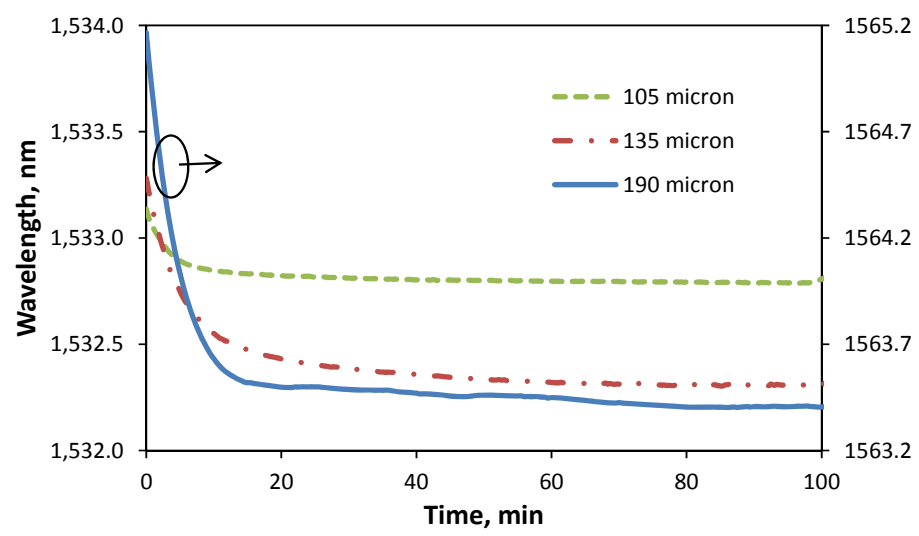

Fig. 7 Time responses of POFBGs with different diameters

The wavelength change of the POFBG is largely introduced by the axial stress due to water induced swelling or shrinking in the fibre. This axial stress is proportional to the longitudinal expansion coefficient of the fibre, which is the contribution of both fibre core and cladding, depending on the material composition and the cross-section area [11]. The recorded time responses of 3 POFBGs with the outer diameters of $105 \mu \mathrm{m}, 135 \mu \mathrm{m}$, and $190 \mu \mathrm{m}$, operated at the same conditions (input optical power, surrounding temperature and humidity), as shown in Fig. 7.

\subsection{Operation in different wavelength bands}

Since vibrational absorption appears as a major source of loss in polymer optical fibres, the fundamental vibration absorption of the molecular bonds mentioned above falls in the infrared region but has absorption overtones extending up to the visible region causing the high optical loss of POFs. The loss spectra of the fibres used were unknown. But it is well known that for the polymer optical fibres the optical absorption loss in $1500 \mathrm{~nm}$ range is much larger than that in visible region [10]. We then investigated the POFBG performance operated in different wavelength bands. 
First we fabricated some POFBG at $\sim 830 \mathrm{~nm}$ using the same type of polymer optical fibre as those used in previous sections. An optical spectrum analyzer was used to monitor the grating reflection. Controlled by a computer the optical spectrum analyzer continuously captured the grating reflection spectrum, tracking the peak wavelength variation of the POFBG staring from the switch-on of a broadband light source centred at $850 \mathrm{~nm}$. A 2-hour tracking didn't find any noticeable change in POFBG wavelength. It was considered that the change in POFBG wavelength over the period was smaller than the analyzer resolution $(50 \mathrm{pm})$.

Another set-up was then designed to look into this issue. We modified the arrangement in Fig.2. Instead of connecting POFBG to port 2 of the circulator, we connected POFBG to port 2 via a WDM fibre coupler. The POFBG with the diameter of $135 \mu \mathrm{m}$ was used for the investigation. We first turned on the broadband source and monitored the time response of the POFBG wavelength until it reached the stabilized value. Then a laser diode was connected to the WDM coupler. Depending on the spectral region of interest, laser diodes with a wavelength of $980 \mathrm{~nm}, 1330 \mathrm{~nm}$ and $1480 \mathrm{~nm}$ were used. This laser introduced additional optical absorption at the specific wavelength, which was also converted into thermal energy and introduce additional wavelength change in the time response of the POFBG used. By setting the output optical power of the laser diode to different levels we can obtain the introduced wavelength change over the input optical power at the laser wavelength. This ratio of wavelength change to input power was measured at 3 different wavelengths as summarized in table 1 .

Table 1 The ratio of wavelength change to input power at different wavelength

\begin{tabular}{|c|c|}
\hline Laser diode wavelength, $\mathrm{nm}$ & Wavelength change/power, $\mathrm{pm} / \mathrm{mW}$ \\
\hline 980 & 5.1 \\
\hline 1330 & 10.9 \\
\hline 1480 & 130.3 \\
\hline
\end{tabular}

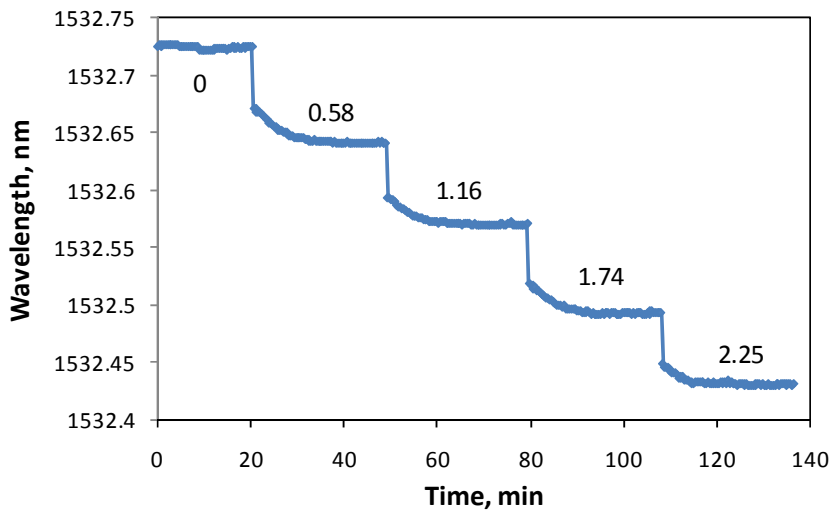

Fig. 8 The wavelength response of POFBG with a diameter of $135 \mu \mathrm{m}$ with input power from a $1480 \mathrm{~nm}$ laser diode varying from $0,0.58 \mathrm{~mW}, 1.16 \mathrm{~mW}, 1.4 \mathrm{~mW}$ to $2.25 \mathrm{~mW}$.

A recoded time response of the POFBG is shown in Fig. 8 while the output power of the laser diode with a wavelength of $1480 \mathrm{~nm}$ was step-changed. The input power to the POFBG at $1480 \mathrm{~nm}$ was measured at the exit of the WDM coupler. When the input power varied from 0 to $2.25 \mathrm{~mW}$ the overall wavelength reduction was $294 \mathrm{pm}$, indicating a ratio of $130.3 \mathrm{pm} / \mathrm{mW}$ at this wavelength. From the summarized results in table 1 for the same input power, the introduced wavelength change at $1480 \mathrm{~nm}$ is much higher than that at $980 \mathrm{~nm}$. This is in agreement with the absorption losses of polymer optical fibres in these regions. 


\section{DISCUSSION AND CONCLUSION}

The wavelength performance of polymer optical fibre gratings has been investigated systematically in this work. Due to the molecular vibration absorption in the fibre part of the optical energy is converted into thermal energy, which changes the water content in the fibre. As a result the characteristic wavelength of a POFBG is changed accordingly. The experiment has shown that the characteristic wavelength of a POFBG starts decreasing after a light source is applied to it. This decrease continues until equilibrium inside the fibre is established. The amount of this wavelength reduction depends on several factors.

First the initial water content inside the fibre, which is related to the surrounding humidity, will have much influence on the final stabilised wavelength. More water content means possible larger wavelength reduction. The introduced wavelength change by the same optical power applied is much larger at $90 \%$ humidity than at $20 \%$ humidity. According to this tendency we expect that the introduced wavelength change may be negligible when POFBGs operate in a very dry environment. The input optical power to POFBG plays an important role in the wavelength response of POFBG. Our work indicates that higher input power introduces larger wavelength change. In fact more input power means more absorption thus more converted thermal energy applied to the fibre. The results in our work also show that POFBGs with different diameters experience different wavelength changes. Our investigation show that POFBGs operating at shorter wavelength experience a smaller wavelength reduction; operating at around $850 \mathrm{~nm}$ POFBGS show a negligible wavelength change.

In general the wavelength change introduced by this optical absorption effect depends on the water content in the fibre, which can be affected by the fibre size, surrounding humidity, input optical power, and absorption coefficient. It can also be affected by the diffusion coefficient of the fibre, which is determined by the composition of the fibre [5]. The time response of the POFBG wavelength can be approximated by an exponential function as the water sorption and desorption in POF can be described by the diffusion theory of mass transfer [9]. Normally the larger introduced wavelength change takes a longer equilibrium time. However, there are some irregularities where some POFBG with a smaller diameter possess a long equilibrium time. This is considered to do with the changed diffusion coefficient in the etched fibre (in our work the fibres with different diameters were achieved by acetone etching). The diffusion coefficient seems having been changed by etching, which may have introduced the irregularity in equilibrium time. A more detailed analysis can be found in our previous work [12].

In conclusion POFBGs will have a stable characteristic wavelength after an equilibrium time. To minimize this internal change caused by absorbed optical energy POFBGs need to operate in dry environment, or be powered by a weak light source, or operate in a short wavelength region.

\section{REFERENCES}

[1] Giaretta, G., White, W., Wegmuller, M., and Onishi, T., "High-speed (11 Gbit/s) data transmission using perfluorinated graded-index polymer optical fibres for short interconnects $(<100 \mathrm{~m})$," IEEE Photon. Tech. Lett., 12(3), 347-349 (2000).

[2] Xiong, Z., Peng, G. D., Wu, B., and Chu, P. L., "Highly tunable Bragg gratings in single-mode polymer optical fibres," IEEE Photon. Tech. Lett., 11(3), 352-354 (1999).

[3] Dobb, H., Webb, D. J., Kalli, K., Argyros, A., J. Large, M. C., and van Eijkelenborg, M. A. , "Continuous wave ultraviolet light-induced fibre Bragg gratings in few- and single-mode microstructured polymer optical fibres," Opt. Lett., 30, 3296-3298 (2005)

[4] Comyn, J., [Polymer Permeability], Elsevier Applied Science Publishers, London, (1985).

[5] Turner, D. T., "Polymethyl methacrylate plus water: sorption kinetics and volumetric changes," Polymer, 23(2), 197-202 (1982)

[6] Harbach, N. G., "Fibre bragg gratings in polymer optical fibres." PhD thesis Lausanne: EPFL (2008).

[7] Zhang, C., Chen, X., Webb, D. J., and Peng, G.-D., "Water detection in jet fuel using a polymer optical fibre Bragg grating", Proc. SPIE, vol. 7503, 750380-1 (2009) 
[8] Thomas, A. M., "Moisture Permeability, Diffusion and Sorption in Organic Film-Forming Materials," Journal of Applied Chemistry, 1(1), 141-149 (1951)

[9] Crank, J., [The Mathematics of Diffusion]. 2nd ed., Clarendon Press - Oxford, (1975).

[10] Kaino, T., [Polymer Optical Fibres - Polymers for Lightwave and Integrated Optics], Marcel Dekker, Inc., New York, 1-38 (1992).

[11] Kronenberg, P, Rastogi, P. K., Giaccari, P., and Limberger, Hans G., "Relative humidity sensor with optical f iber Bragg gratings", Opt. Lett., 27(16), 1385-1387 (2002)

[12]Zhang, W., Webb, D. J., Peng, G.-D., "Investigation into Time Response of Polymer Fibre Bragg Grating Based Humidity Sensors," IEEE J. Lightwave Tech 30(8), 1090 - 1096 (2012) 